\title{
IMPACT OF PHARMACIST LED ANTICOAGULATION MONITORING AND PATIENT EDUCATION ON ORAL ANTICOAGULATION THERAPY WITH ACENOCOUMAROL
}

\section{POOJITHA REDDY CHEDEPUDI ${ }^{1}$, OMSRI MOUNIKA ${ }^{1}$, GADDAM CHANDRIKA ${ }^{1}$, ALDRICH FRANKLIN ${ }^{1}$, RAMESH M $^{2}$ VANITHA RANI N ${ }^{1 *}$, THENNARASU $\mathbf{P}^{1}$}

${ }^{1}$ Department of Pharmacy Practice, Faculty of Pharmacy, Sri Ramachandra University, Porur, Chennai, Tamil Nadu, India. ${ }^{2}$ Department of Accident \& Emergency Medicine, Sri Ramachandra University, Porur, Chennai, Tamil Nadu, India. Email: vanithak9@rediffmail.com

Received: 02 December 2016, Revised and Accepted: 04 July 2017

ABSTRACT

Objective: Acenocoumarol is anoral anticoagulant with narrow therapeutic index, and the ideal dose of anticoagulation lies between International normalized ratio (INR) values of 2.0 and 3.5. Lack of monitoring and knowledge on acenocoumarol therapy may compromise patient's safety resulting in under- or over-anticoagulation. A study was conducted to monitor for the safety of anticoagulation therapy, achievement of goal INR levels and to assess the impact of pharmacist led anticoagulation monitoring and patient education on the rational use of acenocoumarol in patients admitted in cardiology wards with thromboembolic disorders.

Methods: The study was conducted in 70 patients; data collection was done, prescriptions were analyzed for drug-drug interactions and adverse drug reactions (ADRs). Patients' knowledge on acenocoumarol therapy was assessed using a self-developed questionnaire at the baseline, then were subjected to a detailed patient education and on an average each patient received three sessions of education. They were again made to answer the same questionnaire on the day of discharge. The mean score of the responses before and after education was compared statistically using Wilcoxon signed rank test and McNemar test.

Results: There were $60 \%$ patients under anticoagulated with the INR range of $>2.0 .123$ drug interactions were observed in 58 patients, on an average of 2 interactions per prescription, but no ADRs were observed. 59 patients (84.3\%) showed significant overall improvement in knowledge on anticoagulation therapy following education.

Conclusion: This study implies the role of clinical pharmacists in achieving better clinical outcomes in patients receiving oral anticoagulation therapy with acenocoumarol.

Keywords: Acenocoumarol, Anticoagulation, Patient education.

(C) 2017 The Authors. Published by Innovare Academic Sciences Pvt Ltd. This is an open access article under the CC BY license (http://creativecommons. org/licenses/by/4. 0/) DOI: http://dx.doi.org/10.22159/ajpcr.2017.v10i10.16459

\section{INTRODUCTION}

Oralanticoagulants have proved their efficacyin treatmentor prophylaxis of pulmonary embolism, deep vein thrombosis, stroke, intracardiac clots, and atrial fibrillation for the past 60 years [1]. Worldwide, till today, the most commonly used Vitamin $\mathrm{K}$ antagonists were coumarin analogues warfarin and to a lesser extent acenocoumarol [2]. The coumarin analogues are characterized by low therapeutic index and interindividual treatment response variability. Hence, the dosage must be individualized and tapering of dosage is recommended before discontinuation [3]. Oral anticoagulants are associated with an increase hemorrhagic risk despite well-proven efficacy which can limit their use in older people. Warfarin and acenocoumarol work by reducing the effects of Vitamin $\mathrm{K}$ that is used by the body in the process of bloodclotting. It inhibits Vitamin $\mathrm{K}$ reductase resulting in depletion of the reduced form of Vitamin K [4].

Depending on the clinical indication, the optimal dose of anticoagulation or therapeutic range to be aimed at generally lies between International normalized ratio (INR) values of 2.0 and 3.5. Higher INR values up to 4.5 may be required in individual cases [5]. The INR, a standardized measure used for reporting prothrombin time, is the recognized standard for monitoring oral anticoagulation therapy. All the available oral anticoagulants (warfarin, acenocoumarol and phenindione) have narrow therapeutic index, so monitoring is essential to ensure safety and effectiveness. Patient education and cooperation are vital during anticoagulation. A lack of knowledge about the therapy may compromise a patient's safety resulting in: Under- or over-anticoagulation; concurrent self-administration of drugs that may interfere with treatment; a failure to recognize problems, such as bleeding, early; and the inability to manage missed doses $[4,6]$.

The safe management of oral anticoagulation in patients is a challenge in the hospital and the community. The clinical pharmacists with experience can play an important role in inpatient oral anticoagulation management, thereby providing improved cost-effective quality of care. The pharmacist-managed anticoagulation program compared to usual care by the physicians achieved significantly better INR control, and the values were kept in between the range [7].

Various studies have reported the utilization patterns of warfarin as anticoagulation therapy. However, the use of warfarin has declined with the increase in the usage of acenocoumarol, whereas the extent of use of acenocoumarol has not been much reported in the literature [8]. Hence, an attempt was made to assess the drug utilization patterns of acenocoumarol in procoagulant disorders and the extent of patient's knowledge and their medication adherence patterns to oral anticoagulation therapy.

\section{METHODS}

A prospective observational study was conducted in a tertiary care hospital for 6 months. The study included 70 patients between the age 
groups of 21-75 years of both genders who could understand and speak English or local language.

The study was conducted after obtaining the approval of the institutional ethics committee (Ethics number CSP/14/OCT/37/213) and the consent of the study participants. Data on patient's demographics (age, sex, educational status, and economic status) chief complaints, past medical and medication history, lab investigations (prothrombin time, INR), diagnosis, comorbidities, and coprescribed drugs were collected by direct history interview of the patients and from patients' case sheets and documented in the proforma specially designed for the study. The prescriptions of the patients were assessed for the prescribing patterns of acenocoumarol, drug-drug interactions using Stockley's drug interactions [9] and up to date database [10] and the causality of adverse drug reactions (ADRs) were identified using Naranjo's causality assessment scale [11].

All the interactions were categorized as risk A,B,C,D and X indicating there was no known interaction, no action needed, monitoring of therapy, modification of therapy and death, respectively. The educational status and socioeconomic status were classified using Kuppuswamy scale into four classes namely Class I as illiterates; Class II as higher secondary, Class III as graduate, and Class IV as post graduate for the educational status; Class I as upper class, Class II as upper middle, Class III as lower middle, and Class IV as lower for economic status [12].

The study patients were assessed for their knowledge on acenocoumarol therapy using a questionnaire specially developed for the study. The questionnaire consisted of eight questions, and the responses were graded quantitatively as 1 for "Yes" response and 0 for "No" response. The patients were made to answer the questionnaire at the baseline and were subjected to a detailed patient education both verbally and by provision of patient education leaflets prepared specially for the study in English and vernacular language Tamil. Each education session lasted for around 30 minutes and was done till discharge (3-5 days). The patients were again made to answer the same questionnaire after the education session on the day of discharge. On an average, the patient received three sessions of education. The mean score of the responses obtained before and after education was compared for assessing the impact of patient education on knowledge of acenocoumarol therapy.

\section{Statistical analysis}

The data obtained were analyzed statistically using SPSS 16.0 version. To describe about the data descriptive statistics frequency analysis, percentage analysis was used for categorical variables and for continuous variables the mean and S.D were used. Wilcoxon signed rank test was used to find the significant difference in knowledge pre- and post-patient education. McNemar test was used and for comparison total score between pre- and post-patient education. In both the above statistical tool, the $\mathrm{p}<0.05$ was considered as significant level. The applicability and reproducibility of the questionnaire were assessed statistically using Cronbach's Alpha and a value of 0.5-0.6 was considered poor and above 0.6 was considered acceptable.

\section{RESULTS}

A to $\mathrm{L}$ of 70 patients of either sex, aged above 21 years and receiving oral anticoagulation with acenocoumarol were recruited from the wards of the cardiology unit. Of these 70 patients, 37 (52.9\%) patients were males and $33(47.1 \%)$ patients were females. The socioeconomic status and the educational status of the study patients were categorized into IV classes as per Kuppuswamy scale. There were $1(1.4 \%)$ patient in Class I (upper); 25 (35.7\%) patients in Class II (upper middle); 21 (30\%) patients in Class III (lower middle) and 23 (32.9\%) patients in Class IV (lower) socioeconomic status. There were 43 (61.4\%) patients in Class I (illiterates), 18 (25.7\%) patients in Class II (higher secondary), 8 (11.4\%) patients in Class III (graduate), and $1(1.4 \%)$ patient in Class IV (post-graduate) educational status.
The most common indication was found to be rheumatic heart disease with or without mitral stenosis in 27 patients, followed by the mitral valve replacement in 21 patients, 8 patients had Coronary artery disease, 7 patients had atrial fibrillation, 2 patients had post atrial septal defects, and 2 patients had deep vein thrombosis. The INR values were within the target range of 2-3.5 for 27 (38.57\%) patients, $1(1.42 \%)$ patient had INR value above 4.0 and $42(60.01 \%)$ patients had INR values below 2.0 .

In this study, of 70 patients, 123 drug interactions were observed in 58 patients, on an average of 2 interactions per prescription. Of 123 interactions, 7 (5.69\%) interactions were under risk category D indicating that modification in the therapy, 59 (47.96\%) interactions were in risk category $\mathrm{C}$ indicating that monitoring the therapy, $50(40.65 \%)$ interactions were in risk category B indicating that no action needed, and 7 (5.69\%) interactions were in risk category A indicating that there was no known interaction. No ADRs were identified in the study population.

Patient's knowledge on acenocoumarol therapy was assessed using a questionnaire specially developed for the study. The reliability of the questionnaire was found to be acceptable as Cronbach's alpha value was found to be 0.6 . The responses before education and after education were obtained. Table 1 explains the responses given by the study population for the questionnaire. Out of 70 patients, 65 responded "Yes" for the reason for which they were admitted in the hospital (Q1) which improved to 70 patients after education. Although there was an increase in the number of respondents, it was not statistically significant $(\mathrm{p}=0.063)$. For the Q2 on the awareness of their diagnosis, 51 responded "Yes" at baseline which improved to 70 patients post education and the improvement was found to be highly significant $(\mathrm{p}<0.0001)$. For the Q3 on awareness about the drugs that they were taking at present, only 6 responded "Yes" at baseline which improved significantly to 47 patients post education $(\mathrm{p}<0.0001)$; for $\mathrm{Q} 4$ about the purpose of taking the drug acenocoumarol 4 patients responded "Yes" at the baseline which significantly improved to 58 patients post education $(\mathrm{p}<0.0001)$; for Q5 stating "in case if your daily acenocoumarol dose what should you do?" 4 patients responded "Yes" at baseline but all 70 patients responded "yes" post education and the improvement was found to be highly significant $(\mathrm{p}<0.0001)$; for $\mathrm{Q6}$ on "what is the purpose of INR testing and how frequently it has to be done?" none responded "Yes" at baseline but it significantly improved to 50 patients post education $(\mathrm{p}<0.0001)$; for $\mathrm{Q7}$ on awareness of the possible side effects of the drug acenocoumarol none responded "yes" at the baseline which improved significantly to 47 patients after education $(\mathrm{p}<0.0001)$; for Q8 stating "Do you know whom to report if at all any side effects are observed?" 8 patients responded "Yes" at the baseline but the responses improved significantly to 70 patients after education $(\mathrm{p}<0.0001)$.

The questionnaire was scored based on the responses given by the patients. A score of 1 was given for a "Yes" response and 0 was given for a "No" response. A mean score of 1-4 depicted "no improvement in knowledge," and a mean score of 5-8 depicted "improvement in knowledge." Of the total study population, 11 (157\%) showed no improvement in knowledge and 59 (84.3\%) showed improvement in knowledge following education.

A comparison was done between age, gender, educational status, and socioeconomic status with respect to the knowledge of patients about acenocoumarol therapy after education as shown in Table 2. There was a statistically significant influence of gender and socioeconomic status toward patients' knowledge on acenocoumarol therapy ( $p=0.016$ and $p=0.004$, respectively), but significant influence was not found with age and educational status toward patients knowledge on acenocoumarol therapy $(\mathrm{p}=0.561$ and $\mathrm{p}=0.114$, respectively).

\section{DISCUSSION}

Over the past 40 years, an outstanding progress has occurred in the field of oral anticoagulation therapy. New anticoagulants have 
Table 1: Assessment of patient knowledge on acenocoumarol therapy

\begin{tabular}{|c|c|c|c|}
\hline \multirow[t]{2}{*}{ Questions } & \multicolumn{2}{|c|}{$\begin{array}{l}\text { Number of patients responded } \\
\text { "Yes" }(n=70)\end{array}$} & \multirow[t]{2}{*}{ Significance $p$} \\
\hline & Pre-education & Post-education & \\
\hline Do you know the name of your disease? & 51 & 70 & $<0.0001^{* *}$ \\
\hline Name the drugs you are taking at present? & 6 & 47 & $<0.0001^{* *}$ \\
\hline Do you know for what purpose acitrom has been given to you? & 4 & 58 & $<0.0001^{* *}$ \\
\hline In case if you miss your daily acitrom dose, what should you do? & 4 & 70 & $<0.0001^{* *}$ \\
\hline What is the purpose of INR testing and how frequently it has to be done? & 0 & 50 & $<0.0001^{* *}$ \\
\hline What are the possible side effects of acitrom? & 0 & 47 & $<0.0001^{* *}$ \\
\hline Do you know whom to report if at all any side effects are observed? & 8 & 70 & $<0.0001^{* *}$ \\
\hline
\end{tabular}

${ }^{* *} \mathrm{~A} \mathrm{p}<0.01$ was considered as highly significant

Table 2: Baseline characteristics versus patients' knowledge on acenocoumarol therapy

\begin{tabular}{|c|c|c|c|}
\hline \multirow[t]{2}{*}{ Characteristic } & \multicolumn{2}{|l|}{ Outcome of education } & \multirow[t]{2}{*}{ Significance $p$} \\
\hline & No improvement (\%) & Improvement (\%) & \\
\hline \multicolumn{4}{|l|}{ Age range } \\
\hline $41-60$ & $4(36.4)$ & $27(45.76)$ & \\
\hline$>60$ & $3(27.3)$ & $21(35.59)$ & \\
\hline \multicolumn{4}{|l|}{ Gender } \\
\hline Male & $7(63.6)$ & $30(50.84)$ & 0.016 \\
\hline Female & $4(36.4)$ & $29(49.15)$ & \\
\hline \multicolumn{4}{|l|}{ Educational status } \\
\hline Class I (illiterate) & $5(45.5)$ & $38(64.4)$ & 0.114 \\
\hline Class II (higher secondary) & $3(27.3)$ & $15(25.42)$ & \\
\hline Class III (graduate) & $2(18.2)$ & $6(10.16)$ & \\
\hline Class IV (post graduate) & $1(9.1)$ & $0(0)$ & \\
\hline \multicolumn{4}{|l|}{ Socioeconomic status } \\
\hline Class I (upper) & $1(9.1)$ & $0(0)$ & $<0.01^{* *}$ \\
\hline Class II (upper middle) & $4(36.4)$ & $21(35.59)$ & \\
\hline Class IV (lower) & $3(27.3)$ & $20(33.89)$ & \\
\hline
\end{tabular}

**A p value of $<0.01$ was considered highly significant

been introduced, new strategies have been developed for older anticoagulants and new indications have been identified for existing anticoagulants. However, most of the results referred to the treatment with warfarin in literature while acenocoumarol is less studied.

The challenging task of anticoagulation therapy is to attain the therapeutic range of INR with lowest effective dose. Lower than normal INR predisposes patients to thrombotic risk and higher than therapeutic range exposes the patients to potentially life threatening bleeding complications.

Various studies have shown the effectiveness of clinical pharmacist in anticoagulation management both in outpatient and inpatient settings [13]. In this study, acenocoumarol was given to the patients in the brand name of acitrom, at five different doses in $1 \mathrm{mg}, 2 \mathrm{mg}$, $3 \mathrm{mg}, 4 \mathrm{mg}$, and $7 \mathrm{mg}$ once daily for patients with various procoagulant disorders. The INR values were found to be within the therapeutic range of 2.0-3.5 only for 27 patients, whereas 42 patients had an INR of below two indicating that they were under anticoagulated. One patient was given $7 \mathrm{mg}$ of acitrom and the INR value was found to be above eight due to severe hyper anticoagulation. The patient was treated with Vitamin K following which the INR value dropped and then the acitrom dose was tapered to $4 \mathrm{mg}$ at the time of discharge. The study also observed that there was no relation between INR values and doses of acitrom [14].

On comparing the association of acitrom doses with INR values, only 6 patients on $4 \mathrm{mg}$ of acitrom met with mean INR in the target range of 2-3.5. Whereas, patients on other doses of the drug had a mean INR range below target level. The changes in the INR values irrespective of doses could be due to interindividual variability of the patients.
The interaction of oral anticoagulants which coprescribed drugs and the variability in response from different patients makes it very difficult to attain and maintain the therapeutic range of INR. In this study, 123 drug interactions were observed between acenocoumarol and coprescribed drugs. The interaction between acenocoumarol and pantoprazole was found to be highest followed by paracetamol. The majority of interactions require monitoring of therapy whereas interactions with phenytoin, aspirin and naproxen required modification of therapy. Hence, they were brought to the knowledge of the physician. As majority of the patients where under anticoagulated, $(60 \%$ patients had an INR value below the target range of 2.0) no side effects or adverse drug interactions were observed in the study population.

In this study, patients' base line knowledge on oral anticoagulation therapy was found to be low, but significant improvement was observed following patient education. A similar study carried out by Chiquette et al., on "Comparison of an anticoagulation clinic with usual medical care" also stated that an improved anticoagulation control, decreased hemorrhage and thromboembolic events were achieved by the clinical pharmacist managed anticoagulation clinic which in turn reduced hospitalizations and emergency department visits for the patients [15]. A study conducted by Andhuvan et al. emphasized the significant impact of personalized continuous counseling episodes between pharmacist and a patient in improving their medication adherence patterns and promoting rational use of drugs [16].

Oral anticoagulants being high-risk medications that require regular monitoring, continuous multidisciplinary intervention would be very effective in achieving safety and efficacy in patient care. Clinical pharmacists can play a key role in anticoagulation management 
by providing structured patient education to improve medication adherence, regular monitoring of therapy for drug-drug interaction and ADR for making necessary interventions. In accordance to this, the study conducted by Ibrahim et al. also indicated that patients who received intensive counseling from clinical pharmacists showed significantly better understanding than the "usual medical care" group. It is further evident from this study that Pharmacists can play an important role in managing anticoagulation therapy through continuous patient education on appropriate drug use and adherence which will be very effective in minimizing drug-related problems and achieving patient satisfaction [17].

\section{CONCLUSION}

Patients' knowledge on oral anticoagulation drug usage is crucial for innocuous and effective use of oral anticoagulation therapy. Awareness on INR ranges, monitoring requirements, drug - drug interactions and adverse reactions is vital for patients since a positive relationship exists between their knowledge and outcomes of the therapy. The present study highlights the effect of patient education on improvement of patient's knowledge on oral anticoagulation therapy and the overall therapeutic outcome of patients on acenocoumarol. The target range of INR was achieved in $40 \%$ of the patients. The study suggests that a clinical pharmacist driven anticoagulation service can aid patients to achieve a significant therapeutic outcome and also assist the physicians to provide better patient care with added safety.

\section{REFERENCES}

1. Militaru FC, Crisan S, Veda SC, Trifa A, Militaru V, Buzoianu AD. Determinants of hemorrhagic risk during acenocoumarol treatment. Hum Vet Med Int J Bioflux Soc 2012;4(1):23-7.

2. Barcelona D, Vannini ML, Fen L, Balestrieri C, Marongiu F. Warfarin or acenocoumarol: Which is better in the management of oral anticoagulants? Thromb Haemost Schattauer Verlag Stuttgart 1988;80:899-902.

3. Pharmacology of Acenocoumarol. Available from: http://www.uptodate. $\mathrm{com} /$ contents/acenocoumarol-drug-information? source $=$ search result\&search $=$ Acenocoumarol\&selectedTitle $=1 \sim 15$. [Last cited on
2015 Feb 19].

4. Information about Acenocoumarol. Available from: http://www.patent. co.uk/medicine/Acenocoumarol.html. [Last cited on 2015 Feb 19].

5. Indications of Acenocoumarol. Available from: http://www.medindia. net/doctors/drug_information/acenocoumarol.htm. [Last cited on 2015 Mar 07].

6. Use of Acenocoumarol. Available from: http://www.old.health.gov.il/ units/pharmacy/trufot/alonim/4140.pdf. [Last cited on 2015 Mar 07].

7. WeMeReC, Welsh Medicines Resource Centre. Canolfan Adnoddau Moddion Cymru). Monitoring Anticoagulant Therapy in Primary Care. Bulletin 'Independent Prescribing Information for NHS Wales'. March, 2007. Penarth: WeMeReC; 2017. p. 1-6.

8. Hosmane SR, Tucker J, Osman D, Williams S, Waterworth P. Inpatient oral anticoagulation management by clinical pharmacists: Safety and cost effectiveness. J Clin Med Res 2010;2(2):90-2.

9. Young S, Bishop L, Tells L, Dillon C, Hawboldt J, O'Shea P. Comparison of pharmacist managed anticoagulation with usual medical care in a family medicine clinic. BMC Fam Pract 2011;12:88.

10. Drug-Drug Interactions with Acenocoumarol. Available from: http:// www.uptodate.com/home/drugs-drug-interaction. [Last cited on 2015 Mar 12].

11. Naranjo CA, Busto U, Sellers EM, Sandor P, Ruiz I, Roberts EA, et al. A method for estimating the probability of adverse drug reactions. Clin Pharmacol Ther 1981;30(2):239-45.

12. Classification of Educational Status and Socio-Economic Status. Available from: http://www.medind.nic.in/ibv/t09/i12/ibvt09i12p1104. pdf. [Last cited on 2015 Mar 10].

13. Tang EO, Lai CS, Lee KK, Wong RS, Cheng G, Chan TY. Relationship between patient's warfarin knowledge and anticoagulation control. Ann Pharmacother 2003;37:34-9.

14. Lakshmi R, James E, Kirthivasan R. Study on impact of clinical pharmacist's interventions in the optimal use of oral anticoagulants in stroke patients. Indian J Pharm Sci 2013;75(1):53-9.

15. Chiquette E, Amato MG, Bussey HI. Comparison of an anticoagulation clinic with usual medical care: Anticoagulation control, patient outcomes, and health care costs. Arch Intern Med 1998;158(15):1641-7.

16. Andhuvan G, Venkatachalam VV, Sankar V. Impact of pharmacist education on medication adherence in heart failure patients. Int J Pharm Pharm Sci 2014;6(11):474-6.

17. Rana I, Saber-Ayad M. Continuous outpatient warfarin counseling and its effects on adherence. Asian J Pharm Clin Res 2013;6(2):101-104. 